Article

\title{
On the physical nature of the problems of frequency control of asynchronous electric drives
}

\author{
Kodkin Vladimir ${ }^{1}$ \\ 1 South Ural State University, Chelyabinsk, Russia; kodkina2@mail.ru
}

\begin{abstract}
The article proposes to consider the possibility of connecting the problems of engineering synthesis of frequency control systems for asynchronous electric drives with the basic provisions of the theory of identification of asynchronous electric motors based on the equations of a generalized AC electric machine.
\end{abstract}

The article presents the results of experimental studies of load parrying processes in asynchronous electric drives with vector and scalar controls. These results indicate the absence of fundamental advantages as processes in a drive with vector control. This advantage should have manifested itself in a more efficient formation of the moment and fast transients. It is suggested that the reason for this is in too significant errors, assumptions and simplifications of the equations adopted in the derivation of vector control and which are its theoretical basis. A method is proposed for describing asynchronous electric motors by nonlinear transfer functions, which made it possible to formulate the principle of correction of electric drives and a method for assessing their efficiency.

The article shows. that the correction based on the proposed nonlinear transfer functions of the induction motor is much more efficient than the traditional vector control, which was confirmed by detailed experiments and modeling. The results of the most important of which are given in the article. An assumption was made. that the advantage in efficiency is due to a more accurate identification of the dynamics of an asynchronous electric motor with a gear function instead of vector equations.

Keywords: asynchronous electric motor, frequency control, vector control, vector equation, nonlinear transfer functions, experiment

\section{Introduction}

Asynchronous electric drives with frequency control (AED) are a vivid example of the introduction of the most modern scientific and technical achievements into wide practice - in electronics, programming, instrumentation, etc. Despite this, there are very significant "white spots" in the theory of control of such systems, which many experts try not to notice. With the expansion of the use of AEDs in increasingly complex and precise technologies, it becomes more difficult to ignore these problems.

The most widely used are two types of control - "vector" and "scalar" [1, 2, 3, 5, 6, 10-17]. Scalar control, which forms the amplitude and frequency of the stator voltage of an electric motor, is considered by engineers and scientists to be "simple and reliable", but without any possibilities for accurate and fast regulation. To describe the performance in an electric drive with this control method, mechanical characteristics are usually used equivalent circuits and, less often, vector diagrams $[1,2,4]$.

Vector control - aims at direct control of the motor torque, for which rather complex nonlinear calculations are performed in the control unit of the frequency and voltage converter (FC). Therefore, this method is always considered more complex, but effective. Drive equations, which are used to derive control algorithms are generalized equations of asynchronous electric motors with a very large 
number of simplifications and assumptions, the main of which is the assumption of the sinusoidal nature of all currents and magnetic fluxes in the motor. The accuracy of torque control in such electric drives, as follows from experiments of a rather large the number of scientific papers $[5,6,7-9,18,19]$, ultimately, not very high, especially for sensorless regulation (that is, for the vast majority of asynchronous electric drives). There are a huge number of methods of "refinement" of vector control, which FC manufacturers are in no hurry to implement. Moreover, the majority of companies producing frequency converters do not indicate in the documentation the details of "their" control algorithms, and any basic structural solutions. Estimating the error of both methods is very difficult, because in the theoretical provisions on which they are based, a lot of assumptions and simplifications are made.

For several years, the authors of the article carried out detailed experiments on stands with asynchronous electric drives with a power of $3.5 \mathrm{~kW}$ FC - ATV , from «Schneider Electric». The initial goal of the experiments was as follows: to confirm the advantages of vector AED controls over scalar ones and to get recommendations for optimizing the parameters of speed controllers. The main investigated modes were accelerations to various speeds and load surges at these speeds [7, 18-20].

During the research, a number of rather unexpected results were obtained.

\section{Formulation of the problem}

Figure 1 shows the timing diagrams of the processes of regulating the stator currents and the speed of asynchronous electric motors during acceleration (time-1 second) to a speed of $92.4 \mathrm{rad} / \mathrm{s}$ and load surges (interval from 4 to 6 seconds) with a scalar, and vector open-loop speed control. These are examples of bench experiments, which are described in detail in [18-20]. It turned out that it is not easy to see the advantages of a vector control drive over a scalar drive. "Drawdowns" of speed change by no more than $10 \%$, time of transient processes - by $20 \%$. Numerous comparative tests [18, 19] also showed insignificant differences in the main technical characteristics of these control methods.

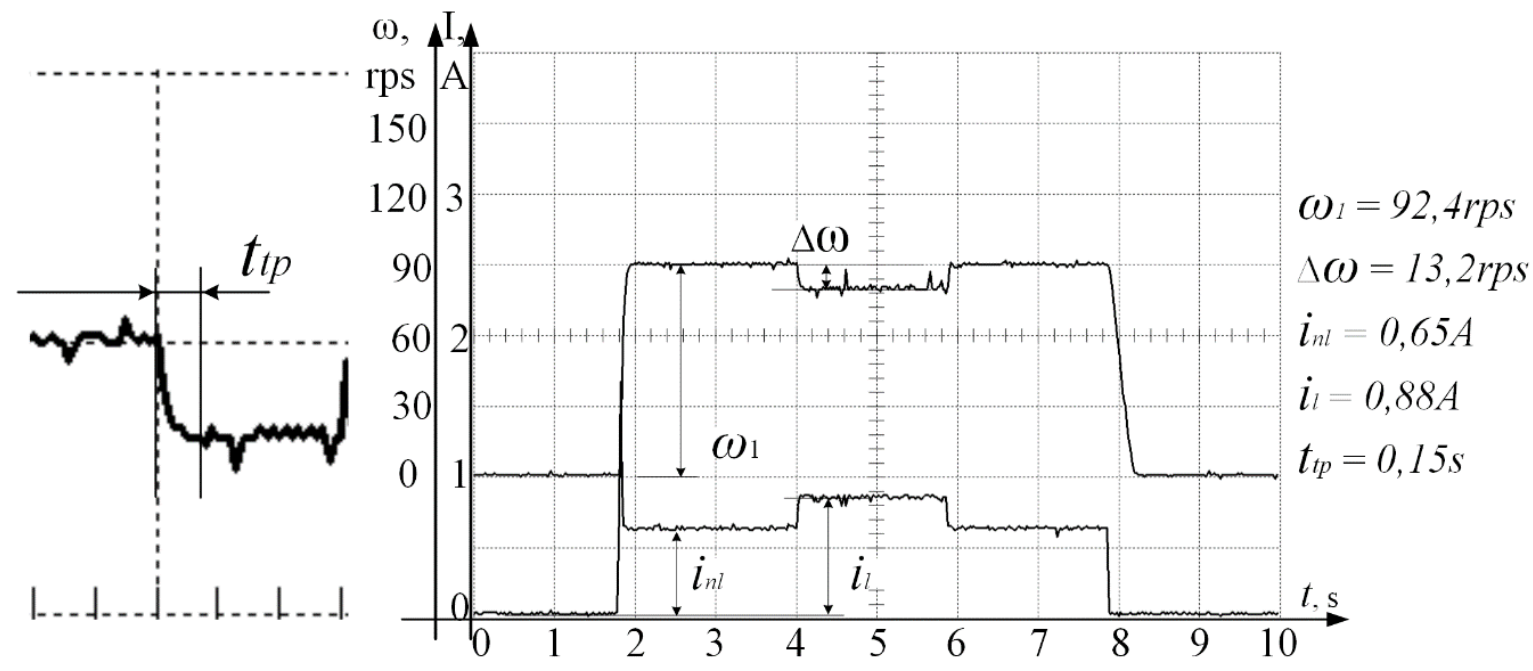

(a) 


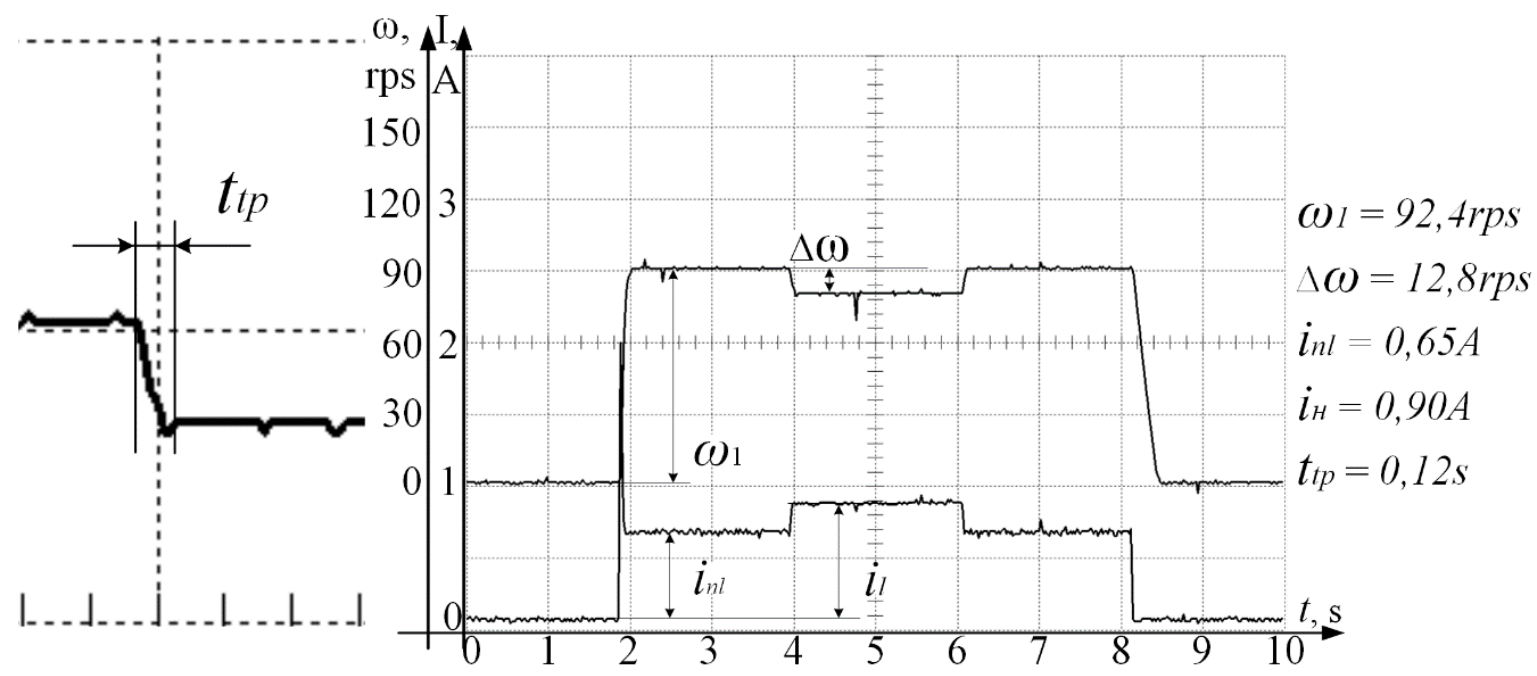

(b)

Figure 1. Diagrams of stator speed and current during acceleration to speed 92,4 rps, in AED with scalar (a) and vector control and with load surge (b).

Figure 2 shows experiments on load parrying in a vector control drive with a PI - speed controller at different speeds. There is also no obvious advantage that could be expected. The static speed error is close to zero. But the time of the transient process - both with load surges and with its removal $1.3 \mathrm{~s}$ (!! ??) instead of $0.1 \mathrm{~s}$ with a scalar one (Fig. 1) - makes this electric drive unsuitable for operation in the vast majority of industrial mechanisms - from lifting transport mechanisms to manipulators.

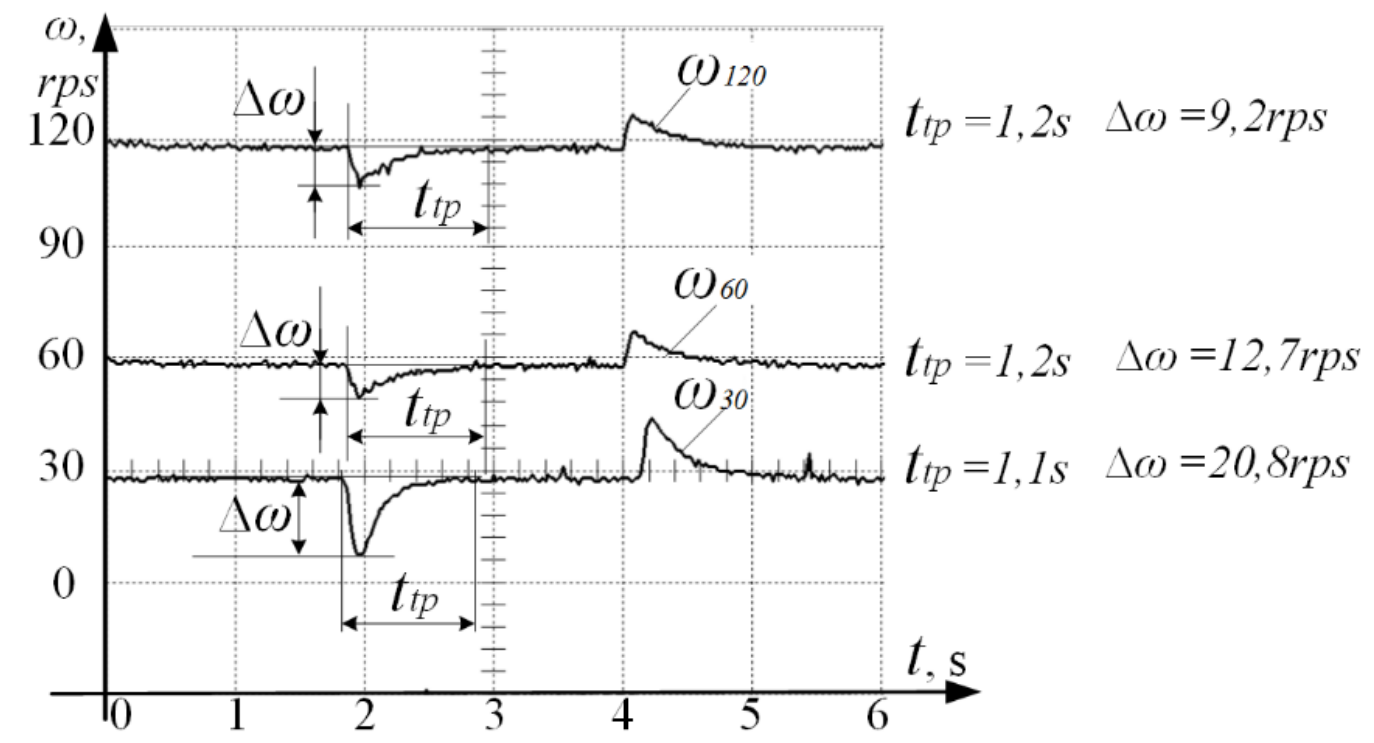

Figure 2. Speed diagrams in asynchronous electric drives with vector control with PI speed controller at three different speeds

It is this and similar experimental results that demanded a rethinking of approaches to

\section{frequency regulation.}

\section{Analysis methods and problems}

Since the 20s of the last century, processes in asynchronous electric motors have been described by generalized equations of an alternating current machine. In engineering calculations, methods 
"derived" from these equations are often used - vector diagrams, equivalent circuits and static (mechanical) characteristics calculated by the Kloss formula. These methods are adapted for frequency control. showing how equivalent circuits or mechanical characteristics change with changes in the frequency of the stator voltage. At the same time, it is neglected that the derivation of all these formulas and characteristics was carried out for a constant value of the stator voltage frequency. For a variable voltage frequency, most conversions would be impossible. It should be noted that it is theoretically very problematic to estimate the error of these transformations.

Meanwhile, in the derivation of both vector and scalar control algorithms, all the equations of variable machines have a number of very controversial mathematical expressions. For example, we will give some equations from the book [1], while immediately it is necessary to stipulate, that all the other books say the same thing.

For scalar and for vector controls, the initial, basic equations are drawn up according to the equivalent circuit of an asynchronous electric motor - formulas (2.10) on page 46 and (2.22) on page 59 , in the book [1].

$$
\begin{gathered}
\text { «...1) } u_{a d}=u_{1}-i_{1} r_{1}=j \omega_{1} \psi_{1} \\
\text { 2) } u_{b d}=u_{1}-i_{1}\left(r_{1}+j x_{1 \sigma}\right)=j \omega_{1} \psi_{m} \\
\text { 3) } u_{c d}=j \omega_{1} \psi_{2} \approx u_{1}-i_{1}\left[r_{1}+j\left(\omega_{1 \sigma}+x_{2 \sigma}\right] \ldots\right. \\
\ldots u_{1 d}=\psi_{2 d} r_{1}\left[T_{2} T_{1}^{\prime} p^{2}+\left(T_{2}+T_{1}^{\prime}+\frac{k_{2} L_{m}}{r_{1}}\right) p+\left(1-T_{2} T_{1}^{\prime} \omega_{1} \omega_{2}\right)\right] / L_{m} \\
u_{1 q}=\frac{\psi_{2 d} r_{1}\left[T_{2} T_{1}^{\prime}\left(\omega_{1}+\omega_{2}\right) p+\left(T_{2} \omega_{2}+T_{1}^{\prime} \omega_{1}+\frac{k_{2} \omega_{1} L_{m}}{r_{1}}\right)+T_{2} T_{1}^{\prime} \omega_{2} p\right]}{L_{m}} \ldots »
\end{gathered}
$$

As follows from the presented formulas, the equations contain terms - the products of inductances and flux linkages, for example - "L" and " $\psi 2 \mathrm{~d}$ " for the frequencies of the stator and rotor currents " $\omega_{1} "$ and " $\omega_{2} "$, both for constant values, which is impossible with frequency control. Thus, the equations that are the basis for the vector control of an asynchronous electric motor are valid only at a constant frequency of the stator voltage (as well as stator and rotor currents) and the harmonic composition of flux linkages, that is, in the complete absence of higher harmonics in the motor currents (!!!).

It is quite simple to make sure that the last assumption does not correspond to reality, in almost any asynchronous electric motor, in any operating mode and any control algorithm. For this, the authors conducted tests, the content and results of which are described in a number of articles $[7,9]$. It is more difficult to estimate the error of such a formulation of the engine equations and the influence of this error on the control efficiency, the algorithm of which is formed according to these equations.

The derivation of the vector control equations contains many simplifications and assumptions, some of which will be mentioned in this work. The studies carried out suggest that the real cost of these inaccuracies is ineffective control of asynchronous electric motors.

It is also obvious that without these simplifications, any engineering analysis and derivation of control algorithms would be extremely difficult. Moreover, most experts consider them inevitable. Let us consider the main, in our opinion, the problems of describing the AED.

1. Sinusoidal currents of the stator and rotor of asynchronous electric motors with frequency control. 
The sinusoidality of the rotor and stator currents is a very important assumption, without which practically the entire mathematical apparatus for describing alternating current electric machines would not have taken place. This assumption is especially "important" for vector control.

Vector control tries to control the torque as the product of flux linkage projections on the longitudinal and transverse axes, formula (2.12) on page 51 in [1]:

$$
m=\frac{3 Z_{p} L_{m}}{2 L_{2}}\left|\psi_{2} \cdot i_{1}\right|=\frac{3 Z_{p} L_{m}}{2 L_{2}}\left(\psi_{2 m} i_{1 n}-\psi_{2 n} i_{1 m}\right)
$$

it is not taken into account. that the flux linkages have higher harmonics, which, in principle, are not controlled, but the selected ones, the fundamental harmonics of the flux linkages, are controlled very indirectly, according to "convenient" coordinates, which are calculated using very simplified formulas, for example, formula (2.23) on page 61, and the diagram on Figure 2.26 on page 62 of the "flux link identifier" in [1]:

$$
\ll \ldots \frac{d \psi_{1}}{d t}=u_{1}-i_{1} r_{1} ; \psi_{2}=\left(\psi_{1}-i_{1} \sigma L_{m} / k_{1}\right) / k_{2}
$$

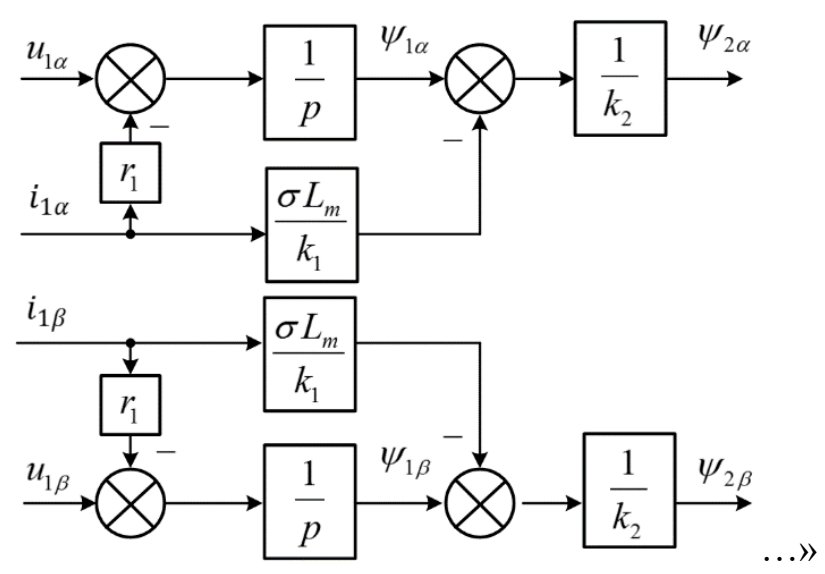

It is very difficult to expect precision and efficiency from such control. But it is difficult to prove this complexity theoretically. Especially quantify it. All this meanwhile has a very serious significance for practice.

(Unlike motors with an active magnetic flux, in which one of the factors in the formula determining the torque is constant, and the higher harmonics of another factor lead to higher harmonics of the torque, which does not affect the operation of the drive, in an induction motor, both magnetic fluxes are periodic - and stator and rotor. Consequently, in the mechanical torque, even from the multiplication of high-frequency components, low-frequency components can be obtained that are completely uncontrolled and uncontrolled. If they form a symmetrical system due to the three phases of the motor, they will only cause heating of the windings, in the worst case - uncontrollable terms of the torque).

In spite of the fact that all FC manufacturers actively offer sets of filters and chokes for frequency converters as filtering external interference, and high frequency interference created by the AED, the theory of AED control allows the neglect of all harmonics of the rotor and stator currents except for the main working one. Of course, the working harmonic of the stator current with a frequency from 
0 to $1000 \mathrm{~Hz}$ (in most FCs) is also capable of suppressing interference, but filters are offered for a much larger range of interference. (?!) While describing the processes of controlling the torque and rotation speed in motors these harmonics are "forgotten".

It is quite easy to check the presence of higher harmonics in the motor currents. These phenomena are most evident in the rotor currents. The experiments are described in detail in [7-9, 1820]. They showed that the rotor currents in static modes also contain significant harmonics that differ from the fundamental. There are enough reasons for their appearance in drives - power elements in the inverter operating in a pulsed mode and, undoubtedly, the variable frequency of the stator voltage.

\section{Imperfection of vector control algorithms}

Most often, considering the dynamics of asynchronous drives, it is vector control that is analyzed, considering that scalar, even corrected, is just a reliable, simple, but inaccurate and slow control, which, in principle, cannot be used in relatively accurate and fast-acting systems. In this case, the problems of vector control are recognized, but are considered to be smaller anyway. The complexity of vector algorithms (the simplest) is too much superior to scalar control.

Usually, vector control problems [10-17, 21-23] are reduced to a mismatch between the motor model in the control unit and the real parameters of the electric motor, which can vary during operation, adding changes in the load torque and other unknown features to this series. Numerous "observers" have been proposed in scientific papers to solve this problem. It is not easy to answer unambiguously how effective they are, but not a single significant manufacturer of FCs has introduced them into circuits and algorithms, obviously, bearing in mind that the vector control itself is already a modal control method, that is, it relies on numerous models calculated with varying degrees accuracy of many unmeasured coordinates.

The purpose of vector control is to reduce the formation of torque to dependencies, close to the dependencies typical for linear control systems (with DC motors). For this, a large number of calculations are performed in the control unit of the frequency converter for nonlinear coordinate transformations and analogs of complex functions that form real processes in the electric motor (flux linkages, for example).

In this case, a lot of inevitable simplifications are used, for example, on page 61, a number of assumptions are made for the coordinate decoupling unit, which calculates the projection of the stator voltage on the $\mathrm{d}$ and $\mathrm{q}$ axes:

"The coordinate decoupling unit can be built on the basis of the equations of the voltagecontrolled IM model (2.22). We can put $\quad \frac{d \psi_{2}}{d t}=0 \quad$ n $\omega_{1}=\omega$ in them... "

Analysis of these equations of a generalized AC electric machine, assumptions and conclusions of control algorithms gradually led to the following questions:

1. How significant are the errors with which the dynamics of processes in the AED are described by a simple consideration of changes in the stator voltage frequency when using mathematical formulas that are accurate only at a constant frequency? 
2. To what extent is it possible to identify a signal with variable frequencies by a vector with variable parameters? Let us recall some theoretical propositions.

A vector is a mathematical element, or a mathematical category that has magnitude and direction, it can describe a sinusoidal process or a signal of only a certain frequency and amplitude. Each such frequency and, accordingly, each stator voltage vector can be assigned its own vector diagram and

If, identifying an asynchronous electric drive with frequency control, we "simply" in the equations obtained for a constant frequency of the stator voltage, put a variable frequency (also with simplifications of the equations), this is equivalent to representing the process in the motor to changes in vector diagrams or equivalent circuits. Moreover, out of all the variety of such schemes and diagrams, our description will take into account only those states. in which currents and voltages will be sinusoidal. That is, separate "spatial" formations are artificially selected from the complex space of such states. Not related to each other. They do not describe intermediate states and transitions between them.

The equations connecting vector quantities cannot take into account the appearance of higher harmonics in the signals of an induction motor and their influence on the operation of an electric drive. They cannot take into account the dynamics of the transition from one structure to another.

As a result, we get a mathematical description of the processes, only in certain modes, close to reality. Such modes can be drive acceleration with a constant dynamic torque. In this case, the assumptions (for example, about the constancy of the rotary magnetic flux) are quite valid. But in other modes - for example - parrying load surges, which "begins" with the braking of the rotor and processes in the rotor, the errors become extremely large. What is the error from replacing dynamic processes with sets of static positions, the more torn between yourself (???) - this is an almost philosophical question and brings us to the time of Zeno, who argued with logical aporias that a continuous process and a sequence of static positions coinciding with a continuous one at specific times - not the same thing. Despite . that engineering problems and methods for their solution are far from philosophical constructions and disputes that took place two and a half thousand years ago - this is an example of how an unsolved philosophical problem is reflected in engineering practice. . The question arises "what is the cost of this neglect?" The provisions presented in this article and their analysis allow us to assert:

The price of these assumptions is the inaccuracy and inefficiency of the vector control of asynchronous electric drives.

On the proposed paradigm in engineering analysis.

It is obvious from the above formulas that the method based on the assumption of the sinusoidality of the motor currents has a significant error and its obvious errors raise many questions. The very first question. Can we call a vector a quantity that continuously changes both amplitude and frequency? What is the phase shift of such a signal? Etc . Let's look at the simplest example.

If the stator voltage changes frequency, then it is usually described by the vector

$$
\bar{U}=A e^{j \omega t}
$$


This corresponds to function from time to time

$$
U=A \cdot \sin \omega t
$$

But with a variable frequency, it should look like this:

$$
U=A \cdot \sin \left(\int_{0}^{t} \omega d t\right)
$$

How to estimate the error in vector interpretation of variable frequency voltage? The problem can be formulated as follows:

1. There is a nonlinear equation that does not have an exact solution for $\varphi_{j}$ - arbitrary functions $\varphi_{j}(x, t)$.

$$
\sum_{i} a_{i} \frac{d^{i} x}{d t^{2}}+\sum_{j} \varphi_{j}(x, t)=0
$$

2. When "freezing" $\varphi_{j}=C_{j}$ - constant values, the solution to the equation is and it is trivial.

$$
X=F\left(a_{i}, C_{j}, t\right)(1 a)
$$

3. When in "defrosting" $\varphi_{j}$ you can get the function $\mathrm{X}$ inaccurate and even erroneous solution for variable function $\varphi$ of time. This is exactly what is done when deriving vector control in [12-4]. Another approach is possible:

4. If you "freeze" the function, you can get the Laplace transform for the original equation

$$
X \sum a_{i} p^{i}+X \sum C_{j} p^{i}=0
$$

And find a solution in the form of the same transformation

$$
X(p)=F\left(a_{i}, C_{j}, p\right)
$$

5. After defrosting the function, the solution turns into a family of solutions - a family of transfer functions:

$$
F\left(a_{i}, \varphi_{j}, p\right)
$$

Question:

-Will this family of solutions according to Laplace: ( 2) and (3) - be "closer" to the real process, which is described by the equation (1), which does not have an exact solution than the defrosted function ( 1a).

That. that in one case it is a function, and in the other it is a family of transformations - evidence that the accuracy of these solutions cannot be the same. If in the first case of an incorrect solution, the nonlinearity of the function is simply "forgotten", then in the second, it "transforms" into the 
multidimensionality of Laplace transformations and transfer functions, remaining an incorrect solution, the qualitative features of the sought functions are preserved and this must be used correctly.

Since this problem belongs to mathematics, the following paradigm is proposed for the engineering solution of the problem.

1. There is a vector control, built on simplifications of the vector equations of an induction motor, described in detail in [1-4].

2. A different identification of the dynamics of the processes in the engine and the correction method "following" from this interpretation are proposed.

3. Compares the efficiency of a vector control drive and the efficiency of a drive corrected by a method based on a different interpretation of the AED. The comparison will be carried out by experimental methods. And the result will confirm the lower error of the method.

\section{SOLUTION Non-linear transfer function}

Like any electromechanical system, an induction motor can be described by differential equations, albeit with substantially non-linear components. When some variables are "frozen", these equations can be linearized. After that, transformations of the equations according to Laplace can be carried out and the transfer functions of the drive links, for example, the links of the formation of the moment [], can be obtained. Changes in the "frozen" coefficients and variables will turn these functions into families of functions. Together they can form families of transfer functions and frequency characteristics .. Unlike the vector equation used by traditional methods for describing asynchronous electric drives, this family will exist for all stator voltage frequencies and describe quite accurately the processes with small changes in the "frozen" parameters. I.e . in contrast to the "discontinuous" space of vector states of a linear object, we will try to get a "filled" continuous, albeit not quite accurate description of the processes in an asynchronous electric motor.

In works [8-10] for the analysis of processes in the AED with frequency control, nonlinear transfer functions are proposed that connect the torque $(\mathrm{m})$, relative slip $(\beta)$ and engine parameters $\left(\mathrm{T}_{2}\right.$ - transient time constant; $\mathrm{Mk}, \mathrm{Sk}$ - critical torque and critical slip, depending on the frequency $\omega 1$ :

$$
m=\frac{2 M_{k}\left(T_{2}^{\prime} p+1\right) S_{k} \beta}{\left(1+T_{2}^{\prime} p\right)^{2} S_{k}^{2}+\beta^{2}},
$$

The transfer function resulting from this dependence connecting the absolute slip and the mechanical moment developed by the AED and the structural diagram:

$$
W(p)=\frac{2 M_{k}\left(T_{2}^{\prime} p+1\right) S_{k}}{\omega_{1}\left[\left(1+T_{2}^{\prime} p\right)^{2} S_{k}^{2}+\beta^{2}\right]}
$$




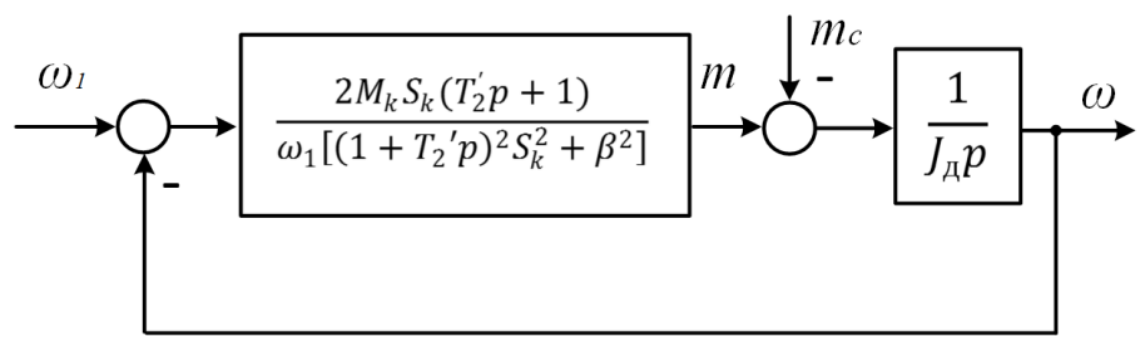

Figure 3.

Where $\omega_{1}$ is the frequency of the stator voltage, and $\mathrm{J}_{\mathrm{d}}$-is the moment of inertia of the motor.

With frequency control, Mk, Sk change - and it is their changes that will show how the W(p) changes with a particular control method. Strictly speaking, this transfer function is also a simplified version of the description of the nonlinear dynamics of an asynchronous electric drive. Variable parameters depending on $\omega_{1}$ and $\beta$ are present in a "frozen" form. It is impossible to obtain such an expression after Laplace transformations (and the transfer function is the result of this very operation). But this formulation of the nonlinear transfer function encompasses the entire state space, describing processes in electric motors, with any spectrum of signals of currents and voltages That is, complex state space, described by an unsolvable nonlinear equation is identified by a nonlinear space, covered by a nonlinear transfer function that exists for any variables and. space is divided into an infinite number of "slices" for any value. And describe the dynamic process in this slice. Since the transfer function formula (4) is continuous. it can be argued, that there are inverse Laplace transforms for signals obtained using this formula, although we cannot get the exact value of these transformations. (to assert the same in relation to the signals obtained from the vector equations, because of their discontinuity).

As follows from formula (4), families of frequency characteristics and transfer functions exist for any value of the frequency of the stator voltage and other signals; moreover, these spectra may not coincide due to the significant nonlinearity of the elements of the frequency electric drive. These are the differences in the interpretation of an asynchronous motor by the transfer function from vector equations and a difference that gives an advantage to this method.

Correction synthesized by a family of transfer functions or frequency characteristics should also be sufficiently accurate and effective.

From this transfer function, methods of correction and evaluation of the effectiveness of the control method follow - according to the magnitude of the absolute slip required to create a torque. These methods are described in article [9].

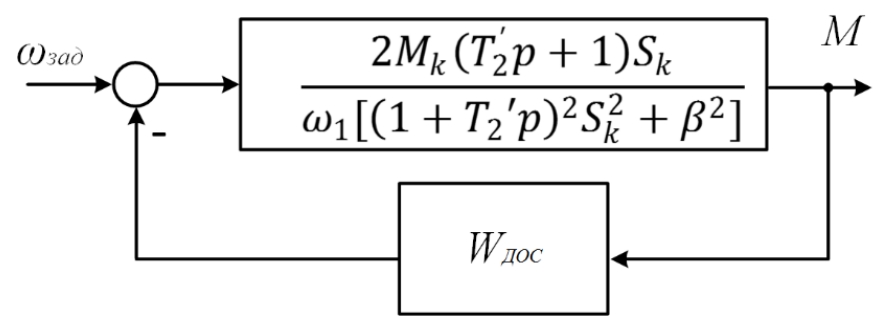

Figure 4. 


$$
W_{\mathrm{DPF}}=-\frac{\omega_{1} \beta^{2}}{2 M_{k} S_{k}\left(T_{2}^{\prime} p+1\right)}, \quad(6) \quad W_{e q u}=\frac{2 M_{k}}{\omega_{1} S_{k}\left(1+T_{2}^{\prime} p\right)}
$$

Figure shows a block diagram of the dynamic positive feedback on the motor torque (DPF + ), which allows you to adjust the family of transfer functions of the torque generator in an asynchronous motor.

\section{Experimental results}

When introducing positive feedback with special dynamic links with a transfer function close to ( 5) into the correction circuits of scalar control, it turned out that it is possible to obtain a stable electric drive with significant advantages over open-loop vector and scalar controls and over closed vector control in terms of speed and accuracy maintaining the speed, which is confirmed by the experiments described in [] and one of the examples of which is shown in Fig. 4. The advantages in terms of transient time and dynamic dips in the speed of a drive with DPF + are obvious in experiments on parrying torque loads - the most difficult operating mode for a drive that maintains rotation speed. The processes are as follows:

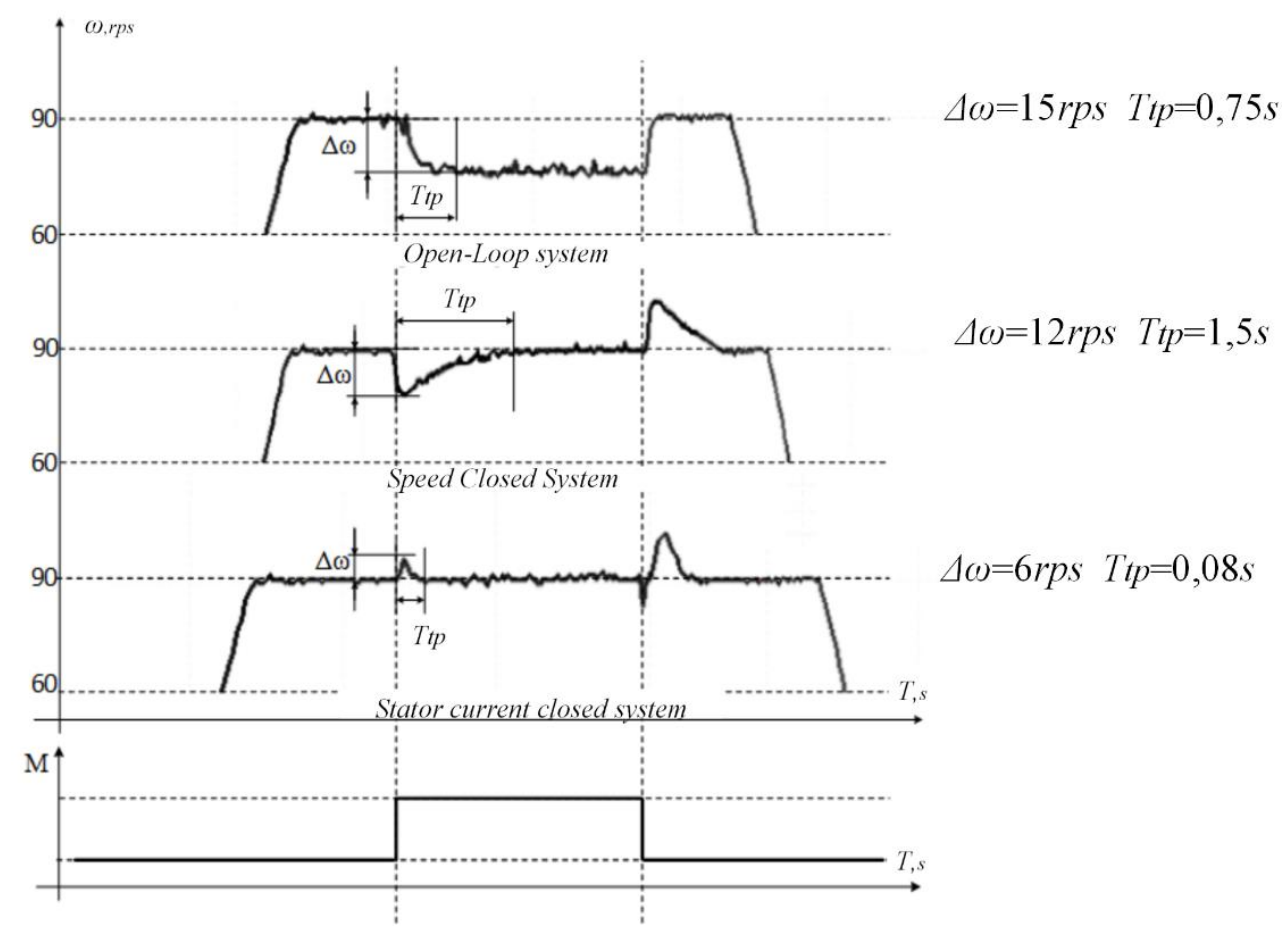

Figure 5.

The main characteristics of the processes: Transient time - $1.5 \mathrm{~s}-$ in a circuit with a PI-vector control regulator and $0.08 \mathrm{~s}$ in a drive with DPF +; Dynamic speed error $12 \mathrm{rad} / \mathrm{s}$ for PI - controller and $6 \mathrm{rad} / \mathrm{s}$ in a circuit with DPF +. Stator static current under load 1.8A - in the drive with PI regulator and $1 \mathrm{~A}$ - with DPF +.

The articles [17-20] describe a method for evaluating the effectiveness of methods for controlling asynchronous electric motors by the frequency of the rotor current, which is rigidly associated with slip. Experiments, described in detail in [ ], have shown that to create the same torque in the motor, corrected by cross-coupling by the torque or by its close analogue - the effective value of the stator 
current - the frequency of the rotor current and, accordingly, the slip is three times less than in a drive with vector control, with a PI regulator $-3.2 \mathrm{~Hz}$ against $9.7 \mathrm{~Hz}-$ fig. 7 .
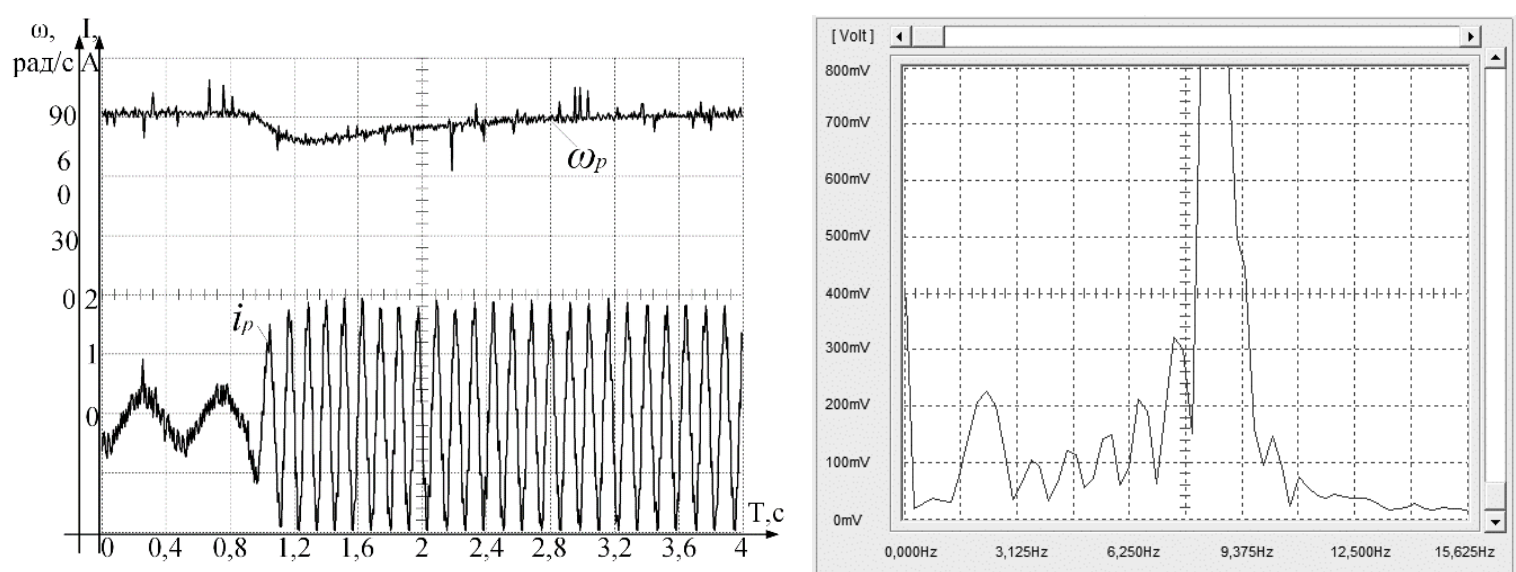

Figure 6. Diagram of the speed and current of the rotor of an asynchronous drive with vector control and the spectrum of the rotor current.
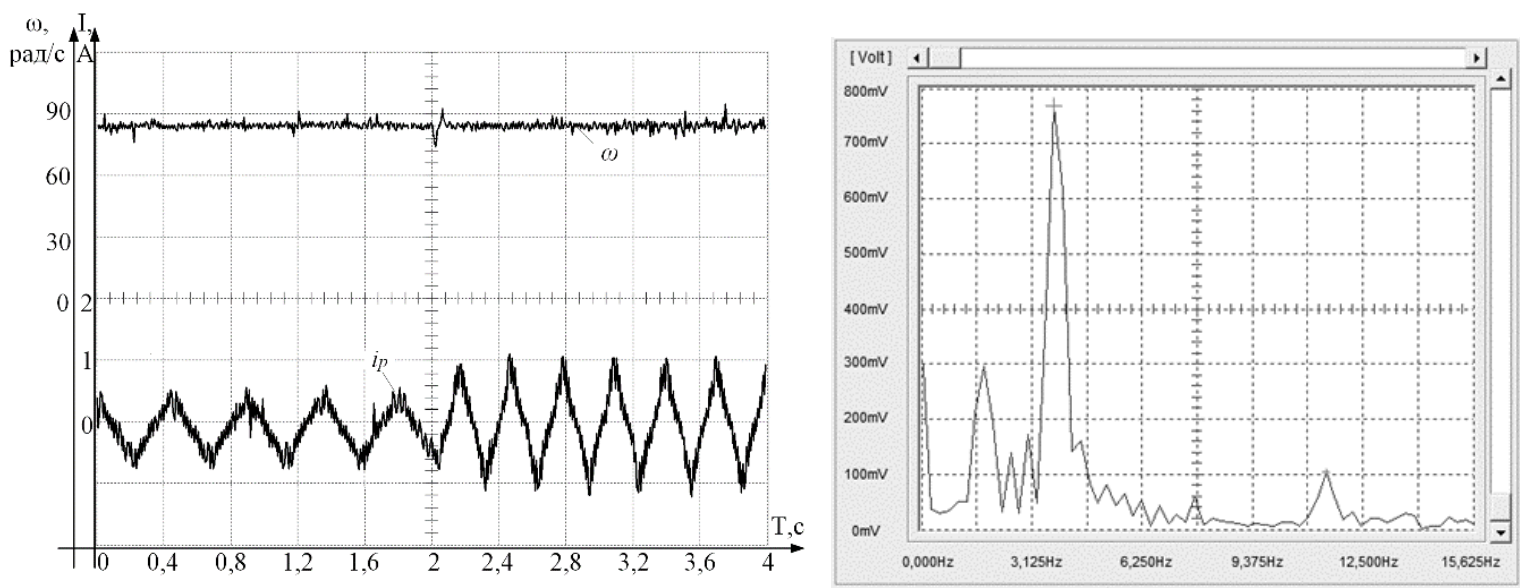

Figure 7. Diagrams of stator speed and current at no-load and load surge in a stator-current-closed system with dynamic link (DPF +) and rotor current spectrum

These experiments showed two main results - the signal spectra of the motor currents with frequency control are nonlinear, the processes themselves are non-sinusoidal, practically under any control. At the same time, for a drive with vector control with a PI - speed controller, the frequency of rotor currents is the highest, that is, to create the same torque in such a drive, the maximum absolute slip of all the considered control schemes is required.

This was also confirmed by numerous experiments and simulations [17-20] of various operating modes of the drive and its standard models. The results are described in detail in articles and reports at scientific conferences. [18-20].

In accordance with the paradigm proposed above, the experimental results allow us to assert that:

-Increased efficiency of the AED with positive feedback analogous to the torque (active component of the stator current) with a dynamic link - this is the price of a more accurate mathematical interpretation of the electric motor and the correction derived from this interpretation.

In other words :

The nonlinear transfer function obtained for variable frequency and slip, although inaccurate, is more accurate than vector equations when describing and correcting processes "inside" each of the 
many nonlinear transfer functions describing the formation of a torque in asynchronous electric motors with frequency control.

\section{Conclusions}

Thus, the experiments confirm the assumption that for a system with signals with variable frequencies, amplitudes and phases, the representation of the system signals by harmonic variables carries a greater error than the representation of the elements of this system by nonlinear transfer functions with "frozen" parameters. This was confirmed by the entire sequence of studies identification of a complex object - a technique for evaluating the slip efficiency necessary to create a torque, determined by the frequency of the rotor current and correction by cross-links - primarily by positive dynamic coupling by the effective value of the stator current, which has shown its effectiveness.

If we analyze vector equations as a technique for describing processes in asynchronous electric drives with frequency control, then it should be noted that these equations relate only static states, and they do not take into account the possible inharmonic composition of signals, therefore, only separate isolated states are taken into account in the "state space" of the electric motor, not interconnected by continuous "surfaces, identifying possible signals of the variables of the electric motor.

A non-linear transfer function, the input of which is absolute slip, depending on the frequency of the stator voltage and load, allows you to obtain an effective way of correcting the drive and ensure the accuracy of maintaining speed during load surges, that is, in the most difficult operating mode for an electric drive. Experiments on such an assessment turned out to be very clear, as well as modeling processes in AED. That is, the linearization of transfer functions according to Laplace has smaller errors than the direct linearization of nonlinear differential equations.

Practical value for a number of drives and industries - including wind turbines, transport, etc. On the basis of this transfer function, correction methods were obtained - a positive cross-connection for the motor torque (in a particular implementation, this connection is for the active component of the stator current) with dynamic links that ensure stability This correction provides significantly better results of work in parrying the load than vector and scalar control, which has been confirmed by experiments and simulations..

\section{References}

1. Usoltsev A.A. Frequency control of induction motors: a tutorial. SPb .: ITMO, 2006, - 94p.

2. Usoltsev, A.A. Vector control of asynchronous motors. Tutorial. - Spb .: ITMO, $2002-120 \mathrm{p}$.

3. Pozdeev, A.D. Electromagnetic and electromechanical processes in frequency-controlled asynchronous electric drives. - Cheboksary: Publishing house Chuvash University, 1998. - 172 p.

4. Park R., Robertson B. The reactances of synchronous machines. // Tr. AIEE, 1928,vol. 47

5. Analysis of the dynamic performance of a variable-frequency induction motor drive using various control structures and algorithms / Alekseev V.V., Emel'yanov A.P., Kozyaruk A.E. // Russian Electrical Engineering. 2016. V. 87. No. 4. P. 181-188. https://link.springer.com/article/10.3103/S1068371216040027

6. Comparative analysis of models for systems of an automated asynchronous drive with vector control / Alekseev V.V., Kozyaruk A.E. // Russian Electrical Engineering. 2013. V. 84. No. 12. P. 702. https://link.springer.com/article/10.3103/S106837121312002X

7. Kodkin V.L. Experimental Research of Asynchronous Electric Drive with Positive Dynamic Feedback on Stator Current // III International Conference on Industrial Engineering, Applications and Manufacturing, ICIEAM 2017 - Proceedings. - 2017. https://ieeexplore.ieee.org/document/8076179/

8. Kodkin, V.L The dynamics identification of asynchronous electric drives via frequency response / V.L. Kodkin, A.S. Anikin, A.A. Baldenkov // International Journal of Power Electronics and Drive Systems.-2019.Vol. 10 No. 1.- P.66-73. http://ijpeds.iaescore.com/index.php/IJPEDS/article/view/15182/10882

9. Kodkin, V.L Performance identification of the asynchronous electric drives by the spectrum of rotor currents / V.L. Kodkin, A.S. Anikin, A.A. Baldenkov // International Journal of Power Electronics and Drive Systems.2019.-Vol. 10 No. 1.- P.211-218.http://ijpeds.iaescore.com/index.php/IJPEDS/article/view/15181/10895 
10. MRAS Speed Sensorless Vector Control of Induction Motor Drives Using Predictive Adaptation Mechanism / Moustafa Zair, Abdeldejbar Hazzab //International Journal of Power Electronics and Drive Systems.-2018.-Vol. 9 No. 4.- P. 1523 1533. http://ijpeds.iaescore.com/index.php/IJPEDS/article/view /13084/11455

11. Yeshin E.K. Electromechanical systems of multimotor drives. Modeling and controlling / Yeshin E.K.Kemerovo: State Technical University of Kuzbass, 2003.- 247 p.

12. Firago B.I. Pavlyachik L.B. Regulating electrical drives of alternating-current.-Misnk: "Technoperspectiva”, 2006. -363 p.

13. Sokolovsky G.G. electrical drives of alternating-current with frequency controlling.-M.: "ACADEMIA», $2006-267$ p.

14. Marc Perron; Hoang Le-Huy Full Load Range Neural Network Efficiency Optimization of an Induction Motor with Vector Control using Discontinuous PWM / Marc Perron; Hoang Le-Huy // 2006 IEEE International Symposium on Industrial Electronics. https://ieeexplore.ieee.org/document/4077917

15. Hoang Le-Huy ; G. Sybille MATLAB/Simulink and PSPice as modelling tools for power systems and power electronics / Hoang Le-Huy ; G. Sybille // 2000 Power Engineering Society Summer Meeting. https://ieeexplore.ieee.org/document/867449

16. Louis-A. Dessaint, Roger Champagne. Real-time simulation of electric drives // Mathematics and Computers in Simulation 63(3-5) $\quad-\quad 2003 \quad-\quad$ pp. $\quad 173-181$. https://www.sciencedirect.com/science/article/abs/pii/S037847540300065X

17. Louis-A. Dessaint, Kamal Al-Haddad. Modelling and Simulation of Electric Machines, Converters and Systems // Mathematics and Computers in Simulation 63(3-5) - 2003 - pp. 135-143.

18. Kodkin V.L. Experimental Research of Asynchronous Electric Drive with Positive Dynamic Feedback on Stator Current // III International Conference on Industrial Engineering, Applications and Manufacturing, ICIEAM 2017 - Proceedings. - 2017. https://ieeexplore.ieee.org/document/8076179/

19. Kodkin V. L., Anikin A. S. Frequency control of asynchronous electric drives in transport //2015 International Siberian Conference on Control and Communications (SIBCON).

20. Kodkin V.L. Spectral Analysis of Rotor Currents in Frequency-controlled Electric Drives / V.L. Kodkin, A.S. Anikin, A.A. Baldenkov // 2nd International Conference on Automation, Mechanical and Electrical Engineering, AMEE 2017 - Proceedings. - 2017

21. Kodkin V.L. The analysis of the quality of the frequency control of induction motor carried out on the basis of the processes in the rotor circuit / V.L. Kodkin, A.S. Anikin, A.A. Baldenkov // XI International Scientific and Technical Conference "Dynamics of Systems, Mechanisms and Machines" - Proceedings - 2017.Omsk, 1416.11.2017

22. Shevchenko, A.F. and Abdel' Maksud Selim, S.A., Vector control of stator's current in synchronous motor with permanent magnets for drowned pump drive,Nauchn. Vestn. Novosib. Gos. Tekhn. Univ., 2011, no. 2(43) 23. Doncker R.D., Pulle D.W.J., Veltman A. Advanced Electrical Drives: Analysis, Modeling, Control / Rik De Doncker, Duco W.J. Pulle, Andre Veltman. Advanced Electrical Drives: Analysis, Modeling, Control. Springer, 2011. - 474 p. 